\title{
The relationship of postoperative electrographic seizures to neurodevelopmental outcome at 1 year of age after neonatal and infant cardiac surgery
}

J. William Gaynor, MD, ${ }^{a}$ Gail P. Jarvik, MD, PhD, ${ }^{\mathrm{b}}$ Judy Bernbaum, MD, ${ }^{\mathrm{c}}$ Marsha Gerdes, PhD, ${ }^{d}$ Gil Wernovsky, MD, ${ }^{\mathrm{e}}$ Nancy B. Burnham, RN, MSN, ${ }^{a}$ Jo Ann D'Agostino, CRNP, ${ }^{c}$ Elaine Zackai, MD, ${ }^{f}$ Donna M. McDonald-McGinn, MS, CGC, Susan C. Nicolson, MD, ${ }^{g}$ Thomas L. Spray, MD, and Robert R. Clancy, MD ${ }^{\mathrm{h}}$

Supplemental material is available online.
From the Divisions of Cardiothoracic Surgery, ${ }^{\mathrm{a}}$ General Pediatrics, ${ }^{\mathrm{c}}$ Psychology, ${ }^{\mathrm{d}}$ Pediatric Cardiology, ${ }^{\mathrm{e}}$ Genetics, ${ }^{\mathrm{f}}$ Cardiothoracic Anesthesiology, ${ }^{\mathrm{g}}$ and Neurology, ${ }^{\mathrm{h}}$ The Cardiac Center at The Children's Hospital of Philadelphia, Philadelphia, Pa, and the Department of Medicine (Medical Genetics), ${ }^{\mathrm{b}}$ University of Washington, Seattle, Wash.

Supported by a grant from the Fannie E. Rippel Foundation, an American Heart Association National Grant-in-Aid (9950480N), and grant HL071834 from the National Institutes of Health.

Read at the Eighty-fifth Annual Meeting of The American Association for Thoracic Surgery, San Francisco, Calif, April 10-13, 2005.

Received for publication April 8, 2005; revisions received May 26, 2005; accepted for publication Aug 8, 2005.

Address for reprints: J. William Gaynor, MD, Division of Cardiothoracic Surgery, The Children's Hospital of Philadelphia, 34th and Civic Center Blvd, Suite 8527, Philadelphia, PA 19104 (E-mail: gaynor@ email.chop.edu).

J Thorac Cardiovasc Surg 2006;131:181-9

$0022-5223 / \$ 32.00$

Copyright () 2006 by The American Association for Thoracic Surgery

doi:10.1016/j.jtcvs.2005.08.062
Objective: The occurrence of a seizure after the arterial switch operation is associated with a worse long-term neurodevelopmental outcome. The significance of seizures after neonatal and infant repair of other congenital heart defects is not known.

Methods: A recent study at our institution demonstrated seizures documented by 48-hour electroencephalographic monitoring in $20(11 \%)$ of 178 neonates and infants after surgery for complex congenital heart defects, including hypoplastic left heart syndrome or variants. The developmental outcomes of this cohort were evaluated at 1 year of age by using the Bayley Scales of Infant Development II, which yields 2 scores: the Mental Developmental Index and the Psychomotor Developmental Index.

Results: Developmental evaluations were performed in 114 (70\%) of 164 survivors, including 36 with hypoplastic left heart syndrome. Postoperative electroencephalographic seizures had occurred in $15(13 \%)$ of 114 of the entire group and in 8 (22\%) of 36 of those with hypoplastic left heart syndrome. For the entire cohort, the Mental Developmental Index was $92.3 \pm 13.5$, and the Psychomotor Developmental Index was $79.9 \pm 18.8$ for patients without seizures, compared with $90.3 \pm 10.7$ and $74.4 \pm 19.3$ for those with seizures (both $P>.5$ ). For the hypoplastic left heart syndrome subgroup, the Mental Developmental Index was $92.3 \pm 14.9$, and the Psychomotor Developmental Index was $74.8 \pm 19.3$ for patients with seizures, compared with $91.9 \pm 12.4$ and $73.9 \pm 18.3$ for those without seizures (both $P>.5$ ). A frontal onset of seizures was predictive of a lower score on the Psychomotor Developmental Index, but not on the Mental Developmental Index.

Conclusions: The occurrence of a seizure after cardiac operation is a marker of central nervous system injury. However, in this cohort of neonates and infants with complex congenital heart defects, the occurrence of a seizure was not predictive of a worse developmental outcome at 1 year of age as assessed by the Bayley Scales of Infant Development II.

$\mathrm{T}$ he occurrence of a seizure in the early postoperative period after repair or palliation of congenital heart defects (CHD) is a marker for a central nervous system (CNS) injury and has been associated with adverse neurodevelopmental sequelae. ${ }^{1-3}$ The research standard for detection and quantification of postoperative seizures remains continuous electroencephalographic (EEG) monitoring. In the Boston Circulatory Arrest Study conducted between 1988 and 1992, continuous EEG monitoring in the first 48 hours after the arterial switch operation for transposition of the great arteries (TGA), with or without a ventricular septal defect (VSD), demonstrated seizures in 27 (20\%) of 136 neonates and infants. ${ }^{1}$ Follow-up 

Abbreviations and Acronyms
$\mathrm{APOE}=$ apolipoprotein $\mathrm{E}$
$\mathrm{CHD}=$ congenital heart defect
$\mathrm{CNS}=$ central nervous system
$\mathrm{CPB}=$ cardiopulmonary bypass
DHCA $=$ deep hypothermia and circulatory arrest
EEG = electroencephalogram
HLHS = hypoplastic left heart syndrome
MDI = Mental Developmental Index
PDI = Psychomotor Development Index
TGA $=$ transposition of the great arteries
VSD $=$ ventricular septal defect

evaluation of the patients in the Circulatory Arrest Study demonstrated that the occurrence of a postoperative EEG seizure was associated with a worse neurodevelopmental outcome at 1 and 4 years of age. ${ }^{2,3}$ A recent study from our institution demonstrated that the frequency of postoperative EEG seizures was less than reported in previous studies. ${ }^{1,4,5}$ EEG seizures were identified in $11.2 \%$ of a heterogeneous cohort of neonates and infants with complex CHDs, including hypoplastic left heart syndrome (HLHS). An increasing duration of deep hypothermia and circulatory arrest (DHCA) was identified as a predictor of seizures. However, the incidence of seizures in children with a limited duration of DHCA was similar to that in infants who underwent continuous cardiopulmonary bypass (CPB) alone. This study was undertaken to evaluate the effect of postoperative EEG seizures on neurodevelopmental outcome at 1 year of age in this cohort.

\section{Patients and Methods Sample}

A subgroup of children enrolled in a prospective study evaluating polymorphisms of apolipoprotein E (APOE) as a risk factor for neurodevelopmental dysfunction also underwent continuous video-EEG monitoring in the early postoperative period. ${ }^{6} \mathrm{~Pa}-$ tients 6 months of age or younger who underwent $\mathrm{CPB}$ with or without DHCA for repair of CHD were eligible. Exclusion criteria included (1) multiple congenital anomalies, (2) a recognizable genetic or phenotypic syndrome other than chromosome 22q11 microdeletions, and (3) a language other than English spoken in the home. The study was approved by the Institutional Review Board at The Children's Hospital of Philadelphia. Informed consent was obtained from the parent or guardian.

\section{Operative Management}

Operations were performed by 5 cardiac surgeons with a dedicated team of cardiac anesthesiologists. Alpha-stat blood gas management was used. Pump flow rates were not standardized for this study. DHCA was used at the surgeon's discretion. Before DHCA, patients underwent core cooling with topical hypothermia of the head to a nasopharyngeal temperature of $18^{\circ} \mathrm{C}$. Modified ultrafiltration was performed in all patients.

\section{Video-EEG Examination}

Details of the video-EEG evaluation have been previously published. ${ }^{5,7}$ Video-EEGs were recorded on 1 of 3 identical dedicated portable Telefactor Millenium Beehive machines (Conshohocken, $\mathrm{Pa}$ ), which capture time-synchronized video images and digital EEG data. A brief 15-minute preoperative baseline study was recorded. Recording was reinitiated after surgery immediately after admission to the cardiac intensive care unit. Video-EEGs were recorded continuously for the first 48 hours after surgery. Studies were terminated only for early death or at parental request. Each record was visually reviewed in its entirety every 24 hours by the recording EEG technologist and independently by a pediatric neurologist. The number of seizures during the study period was recorded. In addition, the sites of origin of the seizures were recorded and classified as frontal or nonfrontal. After confirmation of the presence of seizures, the attending physician was informed of the occurrence of a seizure. All treatment decisions, including the institution of antiepileptic drug therapy, were made at the discretion of the attending physician.

\section{Data Collection}

Preoperative factors including gestational age, birth head circumference, birth weight, Apgar scores, and preoperative intubation were obtained from birth and hospital records. Weight, age at operation, and type of operation were recorded along with perfusion data, including CPB time, aortic crossclamp time, and duration of DHCA. Total support time was calculated as CPB time plus DHCA time. Total DHCA time was calculated as the sum of the duration of each episode of DHCA.

\section{One-Year Neurodevelopmental Examination}

Children were scheduled for their 1-year follow-up visit as they approached 1 year of age. A 2-week window was established so that children were seen at 12 months plus or minus 2 weeks. Children born before 37 weeks of gestation were seen at their corrected age. The developmental assessment was performed first, followed by a medical history and physical and neurologic examinations. The developmental assessment included the Bayley Scales of Infant Development II, which yields scores on 2 indices: the Psychomotor Development Index (PDI) and the Mental Developmental Index (MDI). The medical evaluation included an interim health history and physical examination. The neurologic examination included neuromuscular status, reflexes, and bulbar function. Growth measurements, including weight, length, and head circumference, were obtained. Patients were also evaluated by a genetic dysmorphologist. Chromosomal analysis and testing for microdeletions of chromosome 22q11 were performed as indicated. Recognition of neonatal dysmorphic features may be difficult; therefore, some patients were enrolled in whom the diagnosis of a genetic syndrome was not made until the 1-year evaluation. Results were classified as no definite genetic syndrome or chromosomal abnormality (normal), a definite genetic syndrome or chromosomal abnormality (abnormal), or a suspected genetic syndrome or abnormality (suspect).

\section{Data Analysis and Statistical Methods}

Patients were coded according to a previously described classification that incorporates cardiac anatomy and perioperative physi- 
TABLE 1. Characteristics of patients who returned and those who were alive at 1 year and did not return

\begin{tabular}{|c|c|c|c|}
\hline Variable & $\begin{array}{l}\text { Returned for 1-y evaluation } \\
\qquad(\mathrm{n}=114 \text { ) }\end{array}$ & $\begin{array}{l}\text { Alive, but did not return } \\
\qquad(\mathrm{n}=50)\end{array}$ & $P$ value \\
\hline \multicolumn{4}{|l|}{ Sex } \\
\hline Male & $64(56 \%)$ & $32(64 \%)$ & .35 \\
\hline Female & $50(44 \%)$ & $18(36 \%)$ & \\
\hline Gestational age (wk) & $39(29-41)$ & $39(28-41)$ & .80 \\
\hline Birth weight $(\mathrm{g})$ & $3182(956-4460)$ & $2984(1335-3955)$ & .09 \\
\hline Apgar score at $5 \mathrm{~min}$ & $9(6-9)$ & $9(4-9)$ & .28 \\
\hline \multicolumn{4}{|l|}{ Diagnostic class } \\
\hline 1 & $54(47 \%)$ & $26(52 \%)$ & \\
\hline II & $16(14 \%)$ & $5(10 \%)$ & .88 \\
\hline III & $8(7 \%)$ & $4(8 \%)$ & \\
\hline IV & $36(32 \%)$ & $15(30 \%)$ & \\
\hline Age at first operation (d) & $8.5(1-180)$ & $9(1-188)$ & \\
\hline Aged $\leq 30 \mathrm{~d}$ at first operation & $67(59 \%)$ & $32(64 \%)$ & .26 \\
\hline Number of operations with CPB during first year & $1(1-4)$ & $1(1-3)$ & .169 \\
\hline Number of episodes of DHCA during first year & $76 / 114(67 \%)$ & $30 / 50(60 \%)$ & \\
\hline 1 & $49(64 \%)$ & & .10 \\
\hline 2 & $23(30 \%)$ & $14(47 \%)$ & \\
\hline 3 & $4(6 \%)$ & $16(53 \%)$ & \\
\hline Total support during first year (CPB + DHCA) (min) & $78.5(18-183)$ & $80(28-191)$ & .491 \\
\hline Total DHCA during first year (min) & $45.5(1-97)$ & $61.5(23-134)$ & .88 \\
\hline EEG seizures & $15 / 114(13 \%)$ & $1 / 50(2 \%)$ & .02 \\
\hline
\end{tabular}

$C P B$, Cardiopulmonary bypass; $D H C A$, deep hypothermia and circulatory arrest; $E E G$, electroencephalogram.

ology and that has been shown to be predictive of perioperative mortality. ${ }^{8}$ Class I indicates 2 ventricles with no aortic arch obstruction, class II indicates 2 ventricles with aortic arch obstruction, class III indicates a single ventricle with no arch obstruction, and class IV indicates a single ventricle with arch obstruction. Patients with tetralogy of Fallot and TGA are class I, whereas patients with HLHS and variants are class IV. Data are presented as median (range).

Tests for differences in the demographic characteristics of children who did return for their 1-year follow-up versus those who did not were conducted by using the Student $t$ test for qualitative predictors and $\chi^{2}$ or Fisher exact tests for qualitative predictors for the entire cohort and for the subgroup of patients with HLHS or variants (class IV). The $\chi^{2}$ test was used to test the effects of seizure status on neuromuscular outcome unless cell sizes were less than 5 in a $2 \times 2$ table; then the Fisher exact test was used. To determine whether the number of seizures was predictive of worse outcome, analysis of variance was used to compare data for significant effects of number of seizures on MDI and PDI. The Student $t$ test was used to test the effects of seizure location (frontal vs nonfrontal) on MDI and PDI, and the $\chi^{2}$ test or Fisher exact test was used for neuromuscular outcome. All tests were 2 -sided.

\section{Results}

\section{Study Population}

Between September 16, 2001, and April 2, 2003, 238 eligible infants underwent cardiac operation. Of these, $209(88 \%)$ enrolled in the study of APOE genotype. Continuous post- operative EEG monitoring was performed in 183 (88\%). All patients enrolled in the APOE study were approached for enrollment in the EEG study. Reasons for not undergoing EEG monitoring included parental refusal and unavailability of an EEG monitor.

Complete 48-hour EEG studies were obtained in 178 patients $(97 \%)$. There were 5 hospital deaths $(5 / 178 ; 3 \%)$ and 9 additional deaths $(9 / 173 ; 12 \%)$ before 1 year of age. Of the 164 patients alive at 1 year of age, $114(70 \%)$ returned for evaluation. Characteristics of patients who returned and those who were alive at 1 year and did not return are shown in Table 1. The only significant difference between the 2 groups was that patients with seizures were more likely to return for evaluation.

The study population included 7 patients with TGA, 16 patients with tetralogy of Fallot, 23 patients with VSD (with or without coarctation), 36 patients with HLHS or variants, 7 patients with other forms of functional single ventricle, and 25 patients with a variety of other 2-ventricle cardiac defects. Postoperative seizures had been identified in 15 (13\%) of the 144 patients, including 8 (22\%) of the 36 patients with HLHS or variants. Seizures had not been identified in any of the patients with TGA or VSD and had been identified in only 1 of the patients with tetralogy of Fallot. Seizures had occurred in 2 of 7 patients with other forms of functional single ventricle. The mean number of seizures in the 48-hour study period was $79 \pm 83$, with a 

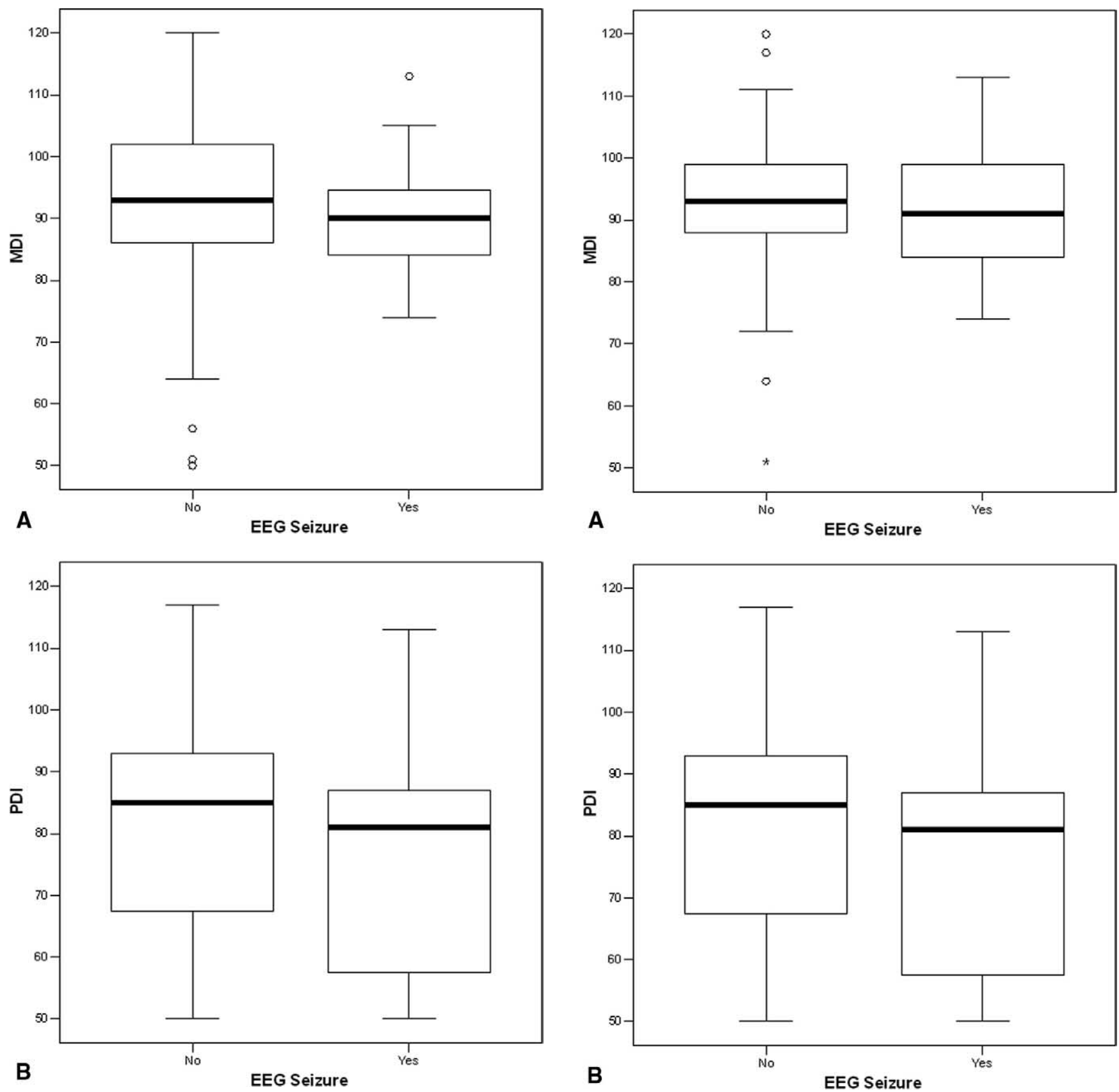

Figure 1. A, Box plot of MDI scores for all patients ( $n=114$ ), stratified by the occurrence of a seizure. B, Box plot of PDI scores for all patients $(n=114)$, stratified by the occurrence of a seizure. Within a box, the solid bar represents the median value, the upper boundary of the closed box represents the 75th percentile, and the lower boundary represents the 25 th percentile. The vertical lines extend to the 10th and 90th percentiles, with outliers plotted as circles.

median of 52 and a range of 1 to 217 . Frontal seizures occurred in $10(67 \%)$ of 15 patients. All patients were treated with phenobarbital after identification of seizure activity. In addition, 3 patients received benzodiazepine therapy.

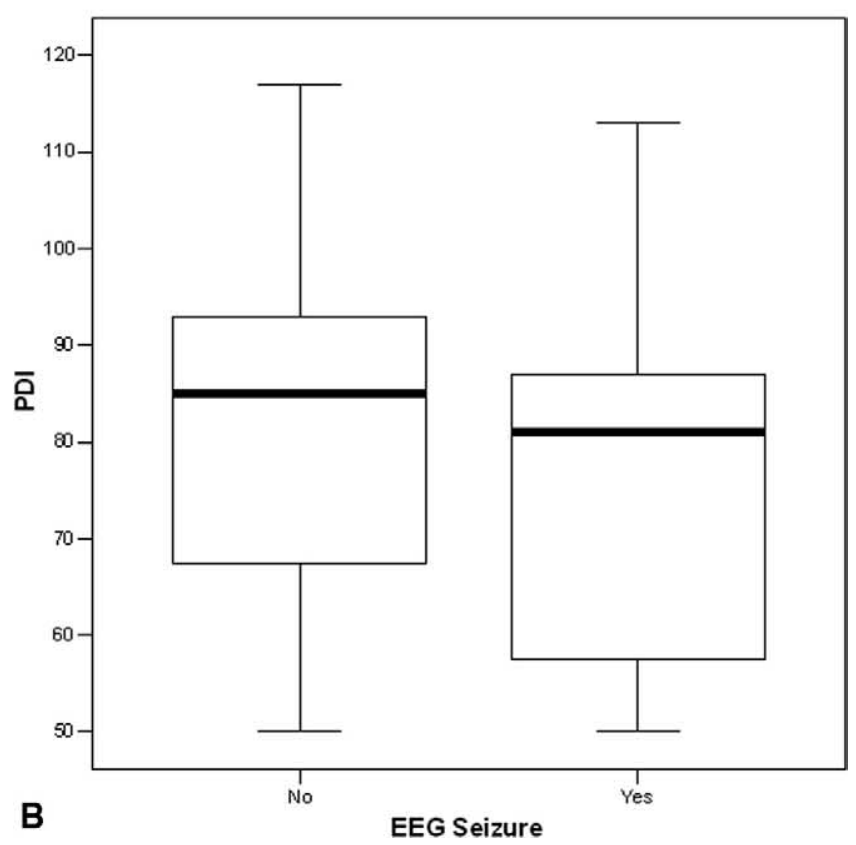

Figure 2. A, Box plot of MDI scores for patients with HLHS or variants $(n=36)$, stratified by the occurrence of a seizure. $B$, Box plot of PDI scores for patients with HLHS or variants $(n=36$ ), stratified by the occurrence of a seizure.

At the 1-year evaluation, the neuromuscular examination results were abnormal or suspect in 52 (46\%) of 114 patients. Abnormal or suspect examination results occurred in $41(41 \%)$ of 99 patients without seizures, compared with 11 (73\%) of 15 patients with postoperative seizures $(P=.027)$. 

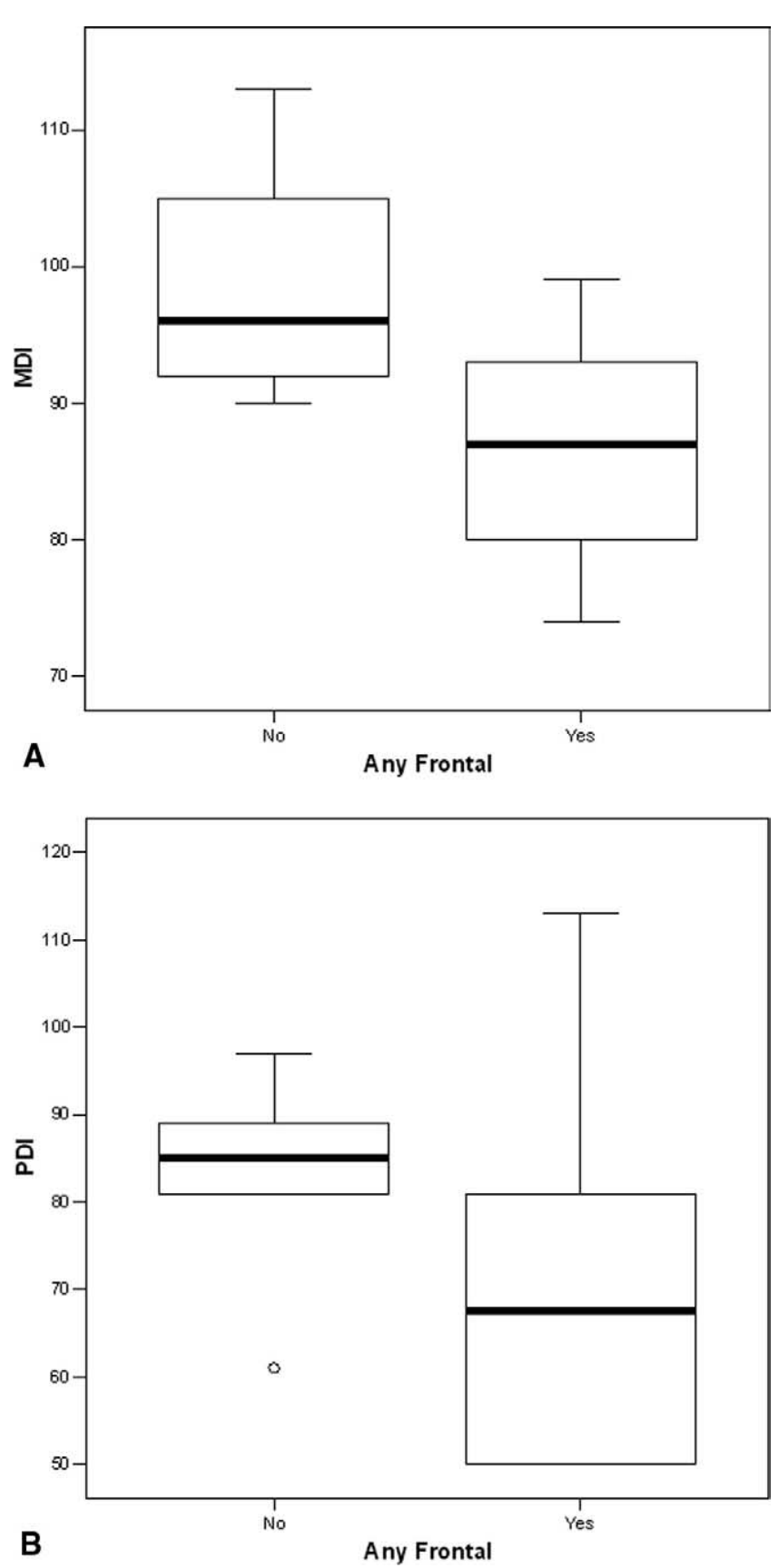

Figure 3. A, Box plot of MDI scores for patients with seizures ( $n=15)$, stratified by frontal or nonfrontal onset of the seizure. B, Box plot of PDI scores for patients with seizures $(n=15)$, stratified by frontal or nonfrontal onset of the seizure.

For the entire cohort, the MDI was $92.0 \pm 13.1$, and the PDI was $79.2 \pm 18.9$. For patients without seizures, the MDI was $92.3 \pm 13.5$, compared with $90.3 \pm 10.7$ for patients with seizures $(P>.5$; Figure $1, A)$. Similarly, the PDI was $79.9 \pm 18.8$ for patients without seizures, compared with
$74.4 \pm 19.3$ for those with seizures $(P>.5$; Figure $1, B)$. Within the subgroups of patients with HLHS or variants, the findings were similar. The MDI was $92.3 \pm 14.9$, and the PDI was $74.8 \pm 19.3$ for patients without seizures, compared with $91.9 \pm 12.4$ and $73.9 \pm 18.3$, respectively, for those with seizures (both $P>.5$; Figure 2). The number of seizures was not predictive of either the MDI or the PDI. However, frontal-onset seizures were predictive of a lower score on the MDI $(P=.03)$, but not on the PDI $(P=.2)$, compared with non-frontal-onset seizures (Figure 3). The frontal onset of a seizure was not associated with increased abnormalities on the neuromuscular examination $(P>.4)$.

\section{Discussion}

The occurrence of a seizure in the postoperative period after a neonatal infant heart operation is a marker of CNS injury. ${ }^{1-4}$ Recent studies at our institution and others have shown that the incidence of seizures in the early postoperative period, as documented by continuous video-EEG monitoring, is less than reported in previous eras. ${ }^{1,5,7}$ Previous studies demonstrated that the occurrence of a postoperative seizure was a predictor of subsequent abnormal neurodevelopmental development. ${ }^{2,3,9}$ In this study, evaluation of children with complex CHDs at 1 year of age after neonatal and infant heart operations suggested that the adverse effect of a postoperative seizure may be less than previously reported. For the entire cohort, there was approximately a 2-point mean decrease in the MDI and a 5-point mean decrease in the PDI, and these decreases were not statistically significant. However, the occurrence of a seizure was associated with a significantly increased incidence of abnormal or suspect neuromuscular examination results.

In the Boston Circulatory Arrest Study, postoperative EEG seizures occurred in $20 \%$ of the patients. Evaluation of the cohort at 1 year of age showed that the occurrence of an EEG seizure predicted a lower PDI score (with a mean decrease of 13 points). ${ }^{2,9}$ Although the MDI scores of children with EEG seizures were lower than those of children without seizures, the differences were not statistically significant. A longer total duration of seizures and seizures with a frontal onset were associated with a trend toward a worse PDI score. ${ }^{2,9}$ Subsequent evaluation of this cohort at 4 years of age demonstrated that the occurrence of an EEG seizure increased the risk of a lower IQ score and increased the risk of abnormalities on neurologic examination. ${ }^{3}$ It is interesting to note that the occurrence of a postoperative seizure was not associated with worse performance on tests of visual and spatial skills. ${ }^{10}$

There are important differences between this study and the Boston Circulatory Arrest Study. The Boston Circulatory Arrest Study evaluated only patients with TGA with or without a ventricular VSD. The current study enrolled children with a variety of complex CHDs, including functional 
single ventricle and HLHS. Children in the Boston Circulatory Arrest Study underwent operation between 1988 and 1992, whereas the children in this study underwent operation between 2001 and 2003. In the interval between the studies, there have been significant changes in prenatal diagnosis; preoperative management; intraoperative management, including the management of bypass and deep hypothermic circulatory arrest; and postoperative care. The Boston Circulatory Arrest Study was a prospective randomized trial that evaluated techniques of CPB and neurodevelopmental outcomes. The current study was subgroup of a prospective observational study that evaluated the effects of APOE polymorphisms on neurodevelopmental outcome. In this study, bypass strategies were at the discretion of the surgeon. It is important to note that investigators in the Boston Circulatory Arrest Study were blinded to the outcomes of the video-EEG and that the children with EEG seizures were not treated with antiepileptic medications (personal communication, Gil Wernovsky, Children's Hospital of Philadelphia, 2005 and Jane Newburger, Children's Hospital Boston, 2005). In the current study, because of the previous finding that the occurrence of a seizure was a risk factor for worse outcome, the physicians caring for the child were notified if video-EEG seizures were identified, and treatment was instituted at their discretion. All of the children in this study in whom a seizure was identified received antiepileptic medications, and this may have decreased the number of seizures and the duration of seizure activity.

The magnitude of the adverse effect of a postoperative seizure in this study was less than that described in the Boston Circulatory Arrest Study. However, as in that study, frontal seizures were associated with a worse outcome compared with nonfrontal seizures. Several factors may have lessened the effect of seizures. Improvements in preoperative and postoperative care may ameliorate the adverse effects of a seizure on the CNS. The patient population in this study was heterogeneous and included patients with complex forms of single ventricle. Many patients required more than 1 operation before 1 year of age. Factors other than seizures may impair neurodevelopmental outcomes in these patients and confound determination of the effects of a seizure. Finally, patients in this study were treated with antiepileptic medications, which may terminate the seizures and lessen secondary CNS injury.

The long-term neurodevelopmental sequelae of postoperative seizures are likely secondary in large part to the underlying CNS injury provoking them. However, recent research in pertinent newborn animal models demonstrates that recurrent seizures induced by proconvulsant drugs that do not otherwise injure the brain may have long-term undesirable effects on brain structure, learning, and susceptibility to spontaneous seizures. ${ }^{11-15}$ Similarly, in other animal models, neonatal seizures create long-term changes in the profile of $\gamma$-aminobutyric acid A subunit composition, the brain's principal inhibitory neurotransmitter receptor site. ${ }^{16,17}$ In addition, phenobarbital administration may be neuroprotective, apart from its antiseizure effects. ${ }^{18} \mathrm{Al}-$ though the major force behind the ultimate neurodevelopmental abnormalities after postoperative seizures may largely be their underlying cause, the magnitude of the contribution of the seizures themselves to the final extent of disability is not known in human neonates.

There are several limitations to this study. Although this cohort was one of the largest to undergo postoperative EEG monitoring and subsequent neurodevelopmental evaluation, the statistical power to discern differences in outcome was limited because of the small number of patients in whom seizures occurred. One-year evaluation was performed in only $70 \%$ of the cohort; however, all but 1 of the surviving seizure patients returned. The Bayley scales provide an excellent assessment of status at 1 year, but their predictive value for neurodevelopmental status at an older age is limited. Children who are at risk for a poor long-term outcome may not be identified on testing at 1 year of age. ${ }^{19}$ More detailed testing of this cohort at an older age may demonstrate differences between the groups. Finally, this was not a treatment trial. Patients were treated with antiepileptic medication after identification of a seizure, but treatment was not standardized, and there was no control group. Therefore, it is not possible to determine conclusively whether treatment affected the neurodevelopmental outcome.

The occurrence of a postoperative seizure is a marker of CNS injury. However, the magnitude of the adverse effect of a seizure on subsequent neurodevelopmental outcome may be less than previously reported. ${ }^{2,9}$ In this study, postoperative seizures were not predictive of a worse outcome at 1 year of age as assessed by the Bayley Scales of Infant Development. However, postoperative seizures were associated with an increase in abnormal findings on the neuromuscular examination. Among the patients with seizures, frontal onset of a seizure was associated with a worse outcome. Treatment with antiepileptic medication may improve outcome; however, further studies are necessary. Evaluation at an older age is needed, and the cohort is currently undergoing a detailed developmental assessment at 4 years of age.

\section{References}

1. Newburger JW, Jonas RA, Wernovsky G, Wypij D, Hickey PR, Karl $\mathrm{CK}$, et al. Comparison of the perioperative neurologic effects of hypothermic circulatory arrest versus low-flow cardiopulmonary bypass in infant heart surgery. N Engl J Med. 1993;329:1057-64.

2. Bellinger DC, Jonas RA, Rappaport LA, Wypij D, Wernovsky G, Kuban KCK, et al. Developmental and neurologic status of children after heart surgery with hypothermic circulatory arrest or low-flow cardiopulmonary bypass. $N$ Engl J Med. 1995;332:549-5.

3. Bellinger DC, Wypij D, Kuban KC, Rappaport LA, Hickey PR, Wernovsky G, et al. Developmental and neurological status of children 
at 4 years of age after heart surgery with hypothermic circulatory arrest or low-flow cardiopulmonary bypass. Circulation. 1999;100:526-32.

4. Clancy RR, McGaurn SA, Wernovsky G, Gaynor JW, Spray TL, Norwood WI, et al. Risk of seizures in survivors of newborn heart surgery using deep hypothermic circulatory arrest. Pediatrics. 2003; 111:592-601.

5. Gaynor JW, Nicolson SC, Jarvik GP, Wernovsky G, Montenegro LM, Burnham NB, et al. Increasing duration of deep hypothermic circulatory arrest is associated with an increased incidence of postoperative electroencephalographic seizures. J Thorac Cardiovasc Surg. 2005; 130:1278-86.

6. Gaynor JW, Gerdes M, Zachai EH, Bernbaum J, Wernovsky G, Clancy RR, et al. Apolipoprotein E genotype and neurodevelopmental sequelae of infant cardiac surgery. J Thorac Cardiovasc Surg. 2003; 126:1736-45.

7. Clancy RR, Sharif U, Ichord R, Spray TL, Nicolson S, Tabbutt S, et al. Electrographic neonatal seizures after infant heart surgery. Epilepsia. 2005;46:84-90.

8. Clancy RR, McGaurn SA, Wernovsky G, Spray TL, Norwood WI, Jacobs ML, et al. Preoperative risk-of-death prediction model in heart surgery with deep hypothermic circulatory arrest in the neonate. J Thorac Cardiovasc Surg. 2000;119:347-57.

9. Rappaport LA, Wypij D, Bellinger DC, Helmers SL, Holmes GL, Barnes PD, et al. Relation of seizures after cardiac surgery in early infancy to neurodevelopmental outcome. Boston Circulatory Arrest Study Group. Circulation. 1998;97:773-9.

10. Bellinger DC, Bernstein JH, Kirkwood MW, Rappaport LA, Newburger J. Visual-spatial skills in children after open-heart surgery. J Dev Behav Pediatr. 2003;24:169-79.

11. Schmid R, Tandon P, Stafstrom CE, Holmes GL. Effects of neonatal seizures on subsequent seizure-induced brain injury. Neurology. 1999; 53:1754-61.

12. McCabe BK, Silveira DC, Cilio MR, Cha BH, Liu X, Sogawa Y, et al. Reduced neurogenesis after neonatal seizures. J Neurosci. 2001;21: 2094-103.

13. Holmes GL, Gairsa JL, Chevassus-Au-Louis N, Ben-Ari Y. Consequences of neonatal seizures in the rat: morphological and behavioral effects. Ann Neurol. 1998;44:845-57.

14. Wasterlain CG. Neonatal seizures and brain growth. Neuropadiatrie. 1978;9:213-28.

15. Yager JY, Armstrong EA, Miyashita H, Wirrell EC. Prolonged neonatal seizures exacerbate hypoxic-ischemic brain damage: correlation with cerebral energy metabolism and excitatory amino acid release. Dev Neurosci. 2002;24:367-81.

16. Zhang G, Hsu FC, Raol YH, Coulter DA, Brooks-Kayal AR. Selective alterations of GABA A receptor subunit expression and function in hippocampal dentate granule cells after seizures in the developing brain. Epilepsia. 2001;42(suppl 7):224.

17. Zhang G, Raol YH, Brooks-Kayal AR. Selective alteration of excitatory and inhibitory receptors and transporters in hippocampal dentate granule cells after seizures in the developing brain. Epilepsia. 2002; 43(suppl 7):27.

18. Hall RT, Hall FK, Daily DK. High-dose phenobarbital therapy in term newborn infants with severe perinatal asphyxia: a randomized, prospective study with three-year follow-up. J Pediatr. 1998;132:345-8.

19. McGrath E, Wypij D, Rappaport LA, Newburger JW, Bellinger DC. Prediction of IQ and achievement at age 8 years from neurodevelopmental status at age 1 year in children with D-transposition of the great arteries. Pediatrics. 2004;114:e572-6.

\section{Discussion}

Dr Richard A. Jonas (Washington, DC). I'd like to begin by congratulating you and your colleagues in undertaking this extremely labor-intensive and very expensive evaluation. I know only too well just how difficult it is to do a study like this. Efforts like yours will certainly help to improve the quality of life of our patients and should be encouraged by all of us.

You made it quite clear in your presentation that you believe that seizures are damaging to the central nervous system, and this is supported by masses of evidence in the neuroscience literature. So why was it not possible for you to demonstrate any impact of postoperative seizures on developmental outcome in this clinical study?

I believe you've begun to answer the question already. Some of the factors you mentioned include, first, that you had a very heterogenous patient population, and many of those-which you did not mention in the presentation but stated in the manuscript I received (thank you)_-did undergo multiple operations, including multiple exposures to circulatory arrest. You also mentioned the fact that seizures that were detected were treated. That is a very important difference from previous studies.

Another important point is that a disproportionate percentage of your patients who returned for developmental assessment had suffered seizures. Those who did not return perhaps had parents who were not worried about their child's developmental progress.

You did mention that your neurological exam did demonstrate a very important impact of seizures on neurological status at 1 year of age. So, clearly, postoperative seizures are not benign.

The interesting questions that are raised in my mind by your study are (1) why did such a high percentage of patients have seizures, and (2) why were your developmental scores so disappointingly low? Eleven percent of your patients suffered seizures. And while this is an improvement over the $20 \%$ of EEG seizures detected in the circulatory arrest study in the 1980s, it is still double the incidence of seizures detected in our prospective randomized trial of $\mathrm{pH}$ strategy that we undertook in the 1990s. The MDI score of your patients was 13 points less than in the $\mathrm{pH}$ trial, and the PDI score, a test of motor function, was 16 points less.

Perhaps, as you implied, this difference can be attributed to different patient populations. In our trials, the majority of patients had transposition, whereas more than a third of your patients had hypoplastic left heart syndrome. But another explanation that I hope you're going to explore might be related to the technique of cardiopulmonary bypass support. Our $\mathrm{pH}$ trial demonstrated that the alpha-stat strategy, which you chose to use in this study, was associated with a higher incidence of morbidity in many different forms; even death achieved a significance level of .06. The publication of that study in 1997 has resulted in the majority of pediatric centers changing to use of the $\mathrm{pH}$-stat strategy. In fact, work published from your own laboratories has demonstrated improved neurological protection with the $\mathrm{pH}$-stat strategy. When I say your own, I mean Dean Kurth's lab at CHOP [Children's Hospital of Philadelphia].

So my first question is, can you please justify your use of the alpha-stat strategy in this trial?

Dr Gaynor. Well, I think if you look at our results, if you look at the patients in this trial who would have met the entry criteria for the $\mathrm{pH}$-stat/alpha-stat study, which were a weight greater than, I believe, 2.2 kilos, no genetic anomalies, and relatively simple straightforward 2-ventricle disease, I looked at that subgroup, and the incidence of seizures in this study, even with alpha-stat, was no different than the incidence of seizures in the $\mathrm{pH}$ arm of your study. So I don't think we can say that in this study it was associated with an increased risk of seizures, at least in comparable patient populations.

I think an important thing to look at is that we did use the Bayley Scales of Infant Development II, which came out in 1993. 
And I know for the circulatory arrest study and, I believe, for the pH-stat study, you used the Bayley Scales of Infant Development I, which was validated in 1969. It's been shown that the performance in those 2 tests is different and that you have to achieve at a higher level on the current test to get the same score.

Dr Jonas. Kids are getting smarter.

Dr Gaynor. Well, basically. But to compare between the 2 scores, you have to add, I believe, 12 points to the PDI score and 11 points to the MDI score, which would explain almost all the differences that we see in mean scores between our groups based on the testing. So I think you can say that our scores are very comparable to the scores that you had.

I think our seizure incidence in comparable patients is exactly the same as in the pH-stat study. And I think the neurodevelopmental study that you showed, looking at the outcomes at 1 to 2 years in the $\mathrm{pH}$-stat study, I believe the conclusion was that neither strategy, neither alpha-stat nor $\mathrm{pH}$-stat, could be statistically associated with either an improvement or decrement in neurodevelopmental outcome as assessed by the Bayley scales. You couldn't identify a statistical difference in outcome.

And indeed, when you looked at the subgroups, there were differential effects. In the tetralogy transposition group, $\mathrm{pH}$-stat was better. However, in the VSD group, alpha-stat children actually performed better.

So I think the data, while I agree with the perioperative data, the data on long-term neurodevelopmental outcomes of alpha-stat versus pH-stat are very mixed. And I don't think there is conclusive evidence that one strategy over the other is associated with improved outcome. That was the conclusion of your paper.

Dr Jonas. Actually the developmental data had a very strong trend in the 2 largest subgroups of patients toward improved outcome with the $\mathrm{pH}$-stat strategy. It was 1 very small subgroup that had a trend in the other direction.

That study in fact confirms the problem of drawing major inferences by using developmental data when you have a heterogenous population in that there is going to be scatter. And perhaps we can explain all of these outcomes on the basis of patient characteristics only. But what I'm suggesting is I don't think this is a hard-enough end point for you to be able to draw a conclusion or even imply that seizures are benign.

But let me go on to my next question. Three years ago I reported at this meeting the results of a prospective randomized trial which demonstrated the importance of perfusate hematocrit in influencing developmental outcome. And in spite of what you and I have just said about the scatter of data with developmental outcomes, that study was shut down by the NIH [National Institutes of Health] because of a powerful impact on the psychomotor development score with an improved outcome with a hematocrit of $27 \%$ versus a hematocrit of $21 \%$.

I was surprised, therefore, that your manuscript makes no mention of the hematocrit that was employed during bypass and does not cite that paper. So can you please describe for us the hemodilution strategy that was employed for your patients?

Dr Gaynor. Actually, because of your study, our hemodilution strategy changed. When we initiated the study, hemodilution to a hematocrit of approximately 25 was achieved in most children. By the end of the study, the average hematocrit after hemodilution was between 29 and 30 . So it actually changed.
We did include hematocrit in the study we presented last year looking at incidence of seizures, and we could not identify an effect of hematocrit on seizures as a risk factor for seizure. But I agree that I think that is a very important study. And we actually changed our management strategy during the course of this study on the basis of your study.

Dr Jonas. Well, in conclusion, I certainly applaud you for undertaking this very difficult work and encourage you to continue to explore means by which we can improve outcomes for our patients. But I do think that it would be a major disservice for our future patients if anyone were to leave here with the impression that EEG seizures are of no consequence and that any difference in development relative to normal is simply a consequence of the presence of a particular form of congenital heart disease. Thank you.

Dr Gaynor. I agree. As we stated up front, I think that occurrence of a seizure is a marker of an injury. However, I think improvements in how we care for the children may have lessened the adverse impact.

But more importantly, I think it may be that our testing at 1 year is very limited, and that's why this entire cohort is currently undergoing evaluation at 4 years of age. And as in the circulatory arrest study, they were still able to demonstrate an adverse effect of a seizure on outcome at 4 years of age. We may be able to demonstrate that at 4 years of age. I don't know yet. But I do agree that a seizure is a marker, and I don't think anybody should go away with the idea that a seizure is a good thing to have. But I'm just saying that it may be that the magnitude of the adverse outcome may be less than previously reported.

Dr Francois Lacour-Gayet (Denver, Colo). You said, in the beginning of your presentation, that there was a correlation between the length of circulatory arrest and the occurrence of seizures. We personally, in Europe and now in the US, are faithful to a technique that tries not to use circulatory arrest at all and have recently used cerebral perfusion for repair of the aortic arch and for the Norwood operation. Basically, the only surgery where I still use circulatory arrest is for repair of total anomalous pulmonary venous return.

Furthermore, there are a number of centers in Japan and Europe that are now doing surgery with patients under normothermia. And of course, all these surgeries are done on full-flow cardiopulmonary bypass, which means a flow that is more than $150 \mathrm{~mL} \cdot \mathrm{kg}^{-1} \cdot \mathrm{min}^{-1}$.

My question is, why do you still use circulatory arrest in most of your patients? And what is the rationale for maintaining this technique of circulatory arrest, as we know that it potentially can hurt the neurological development of the patient?

Dr Gaynor. Circulatory arrest was not used in all these patients. It was used in approximately $40 \%$ of the patients-mainly the patients with hypoplastic left heart syndrome and other complex defects.

But I think it's important, as we introduce new techniques such as regional cerebral perfusion, that you can't just assume that a technique like that is going to result in an improved outcome. I mean, there have been very careful studies, as Dr Jonas mentioned, evaluating circulatory arrest and low flow, blood gas management strategies, and hematocrit with neurodevelopmental outcomes, with seizure monitoring. I think unless you do those types of outcomes, we cannot assume that a new technique has an advantage. It is theoretically attractive, but it may not have an advantage. 
So I think before we can say that these techniques are definitely advantageous, they need to be subjected to the same type of scrutiny other techniques have been in the past.

Dr Antonio F. Corno (Liverpool, United Kingdom). At this point, I have mostly to duplicate what Francois has already said.

I' $m$ impressed by the number of resources that you in Philadelphia, like our colleagues in Boston, are using to study the negative consequence of cardiopulmonary bypass. In Liverpool, we are using a completely different strategy, which is again normothermia, high flow, and almost-normal hematocrit. And this is going to the root of the problem.

I have the impression that you guys are using many resources to study if a brand of cigarette is more or less dangerous than another one, when no smoking is much better. And for no smoking, I mean a strategy of cardiopulmonary bypass much closer to physiology than either deep hypothermia or low flow.

Dr Gaynor. I agree that there may be advantages to high-flow normothermic bypass. There may be advantages to regional cerebral perfusion. But you need to do this kind of study with the EEG monitoring, with neurodevelopmental testing, to show that there is an advantage. There is no other way to show there is an advantage.
Dr Corno. Since we do not have the same resources that you have, why don't you organize a prospective study using deep hypothermia or low-flow versus normothermia with high flow?

Dr Gaynor. That may be a study in the future.

Dr Carl L. Backer (Chicago, Ill). One question that I have is, do you think we should be routinely monitoring all neonates for seizures? I'm impressed that some of your good results are from early diagnosis and therapy of subclinical seizures.

Dr Gaynor. I think that may be. And a treatment trial would obviously be a next step, based on a trial that is actually designed as a treatment trial. I think that, as Dr Jonas mentioned, this is a very time- and money-intensive labor to do these types of monitoring. So I think from studies like this we could probably see, if we look at our TGAs, our TETs [tetralogies of Fallot], and our VSDs, that the incidence of seizure is so low that it's probably not cost-effective.

And again, I don't think I would advocate routine monitoring without setting up a treatment trial of routine monitoring and institutional therapy to look at outcomes. But perhaps in the high-risk subgroups, that would be our next step.

\section{Authoritative}

The Journal of Thoracic and Cardiovascular Surgery is the most frequently cited thoracic/cardiovascular surgery journal in the Science Citation Index. An article in JTCVS is cited on average almost twice as often as those in the closest cardiothoracic journal. 